\title{
REA_10 - Development of a rapid test for detection of anti-COVID-19 IgG and IgM antibodies
}

Thiciany Blener Lopes ${ }^{1 *}$; Fabiana Fioravante Coelho²; Anna Raquel Ribeiro dos Santos ${ }^{3}$; Natália Salazar de Castro 3 ; Flávia Fonseca Bagno'; Ricardo Tostes Gazzinelli"; Ana Paula Salles Moura Fernandes ${ }^{1}$.

${ }^{1}$ UFMG - Universidade Federal de Minas Gerais;

${ }^{2} \mathrm{PMMG}$ - Polícia Militar de Minas Gerais;

${ }^{3}$ UFMG - CT-Vacinas;

${ }^{4}$ Fiocruz/CPqRR.

Introduction: Covid-19 is an infectious disease caused by the SARS-CoV-2 virus that spread to all countries in a short time, acquiring a pandemic character and becoming a public health emergency of international interest. Huge efforts were necessary to develop methodologies for diagnosing the infection. The rapid immunochromatographic test (ICT), a visual test based on the antigen-antibody reaction, has become an interesting alternative given its simplicity, to access anti-SARS-CoV-2 specific antibodies.

Objective: Develop a rapid immunochromatographic test capable of detecting IgM and IgG antibodies against the SARS-CoV-2 virus, with high accuracy.

Methodology: A modified version of the $\mathrm{N}$ (nucleocapsid) recombinant antigen produced by CT VacinasUFMG, a commercial recombinant S (S1 subunit) and the rabbit IgG antibody were conjugated to colloidal gold nanoparticles. The rabbit IgG antibody used as control was obtained from unimmunized rabbits and purified in house. The conjugates produced were dispensed on previously treated fiberglass membranes. Nitrocellulose membranes were impregnated with anti-human IgG and anti-human IgM antibodies to compose the test lines, and anti-rabbit IgG antibody for control line. The membranes were superposed on an adhesive card, cut in $3.2 \mathrm{~mm}$ strips and inserted in a plastic cassette. Several parameters (size of particles, antigen amounts, buffers compositions, among others) were adjusted for better performance. The test was performed by dispensing $10 \mu \mathrm{L}$ of sample (whole blood, serum or plasma), followed by the addition of two drops of running buffer. The result was read after 20 minutes The rapid test developed was evaluated comparatively to TR DPP ${ }^{\circledR}$-COVID-19 IgM/IgG - Bio-Manguinhos using 33 positive samples of whole blood, 47 positive samples of serum/plasma and 47 negative samples of serum/plasma. Positive samples are from patients diagnosed with SARS-Cov-2 by qRT-PCR methodology and negative samples are from health donors.

Results: Whole blood assessments generated a sensitivity of $92.9 \%$ for $\operatorname{IgM}$ detection and $82.6 \%$ for IgG detection. A comparative sensitivity of $96.2 \%$ for IgM and $84.8 \%$ for IgG was obtained for serum and plasma samples. The specificity of the test was greater than $98.0 \%$. Applying the qRT-PCR methodology as the gold standard, the ICT showed a positivity of $89.4 \%, 85.1 \%$ and $97.9 \%$ in the detection of IgM, IgG and IgG or IgM, respectively. These results indicate a superior performance to that found with TR DPP ${ }^{\circledR}-C O V I D-19$ IgM/IgG - Bio-Manguinhos that showed positivity of $55.3 \%, 70.2 \%$ and $72.3 \%$ in the detection of $\operatorname{IgM}$, IgG and IgG or IgM, respectively, compared to the results of qRT-PCR.

Conclusion: The test developed has high sensitivity and specificity for whole blood, plasma and serum samples. The results indicate that the use of two antigens improved sensitivity. Therefore, the test is suitable for use in the field or in laboratory conditions for the serological diagnosis of COVID- 19 .

Keywords: Rapid test; COVID-19; Serological diagnosis 